\title{
Comparaison des ectomycorhizes naturelles entre le hêtre (Fagus sylvatica) et deux lactaires (Lactarius blennius var viridis et Lactarius subdulcis). II. Caractérisation cytochimique des interfaces
}

\author{
JC Pargney, A Prévost \\ Laboratoire de biologie forestière, faculté des sciences, \\ université Henri-Poincaré Nancy-I, BP 239, 54506 Vandœuvre cedex, France
}

(Reçu le 24 mars 1995 ; accepté le 15 mai 1995)

\begin{abstract}
Summary - Comparison of natural ectomycorrhizae between beech (Fagus sylvatica) and two fungi (Lactarius blennius var viridis and Lactarius subdu/cis). II. Cytochemical characterization of interfaces. Fagus sylvatica/Lactarius blennius var viridis and Lactarius subdulcis ectomycorrhizae were studied cytochemically (PATAg test, Gomori-Swift reaction, WGA-colloidal gold labelling) to characterize cell wall and matrix components. Different intensities of reaction were observed between walls of both Lactarius species and according to interfaces in the one mycorrhiza. The mantle-soil interface of $L$ blennius mycorrhizae was characterized by mucilagenous material rich in chitin. $L$ subdulcis mycorrhizae were infected by an ascomycete whose walls were bordered by an electron-dense layer. A decrease of chitin content was observed in the ascomycete walls between the hyphae present in the mantle and intracellularly. In both mycorrhizae, the PATAg test revealed an accumulation of glycogen in rosettes along the plasmalemma of the Hartig net hyphae.
\end{abstract}

ectomycorrhizae / Lactarius / Fagus sylvatica / cytochemistry

Résumé - Des ectomycorhizes de Fagus sylvaticaLactarius blennius varviridis et $L$ actarius subdulcis sont étudiées cytochimiquement (test PATAg, réaction de Gomori-Swift, marquage par le complexe WGA-or colloïdal) pour caractériser les constituants pariétaux et les ciments. Différentes intensités de réaction sont observées entre les parois des deux lactaires et en fonction des interfaces d'une même mycorhize. L'interface manteau-sol de la mycorhize à L blennius se caractérise par la présence d'un mucilage riche en chitine. Les mycorhizes de $\mathrm{L}$ subdulcis sont colonisées par un ascomycète dont les parois sont limitées par une couche dense aux électrons caractéristique. Une diminution de la chitine est observée dans les parois de l'ascomycète entre les hyphes présentes dans le manteau et celles qui envahissent les cellules racinaires. Dans les deux types de mycorhizes, le test PATAg révèle des accumulations de glycogène sous forme de rosette le long du plasmalemme des hyphes du réseau de Hartig.

ectomycorhize / Lactarius / Fagus sylvatica / cytochimie ultrastructurale 


\section{INTRODUCTION}

Le système racinaire du hêtre (Fagus sylvatica L) porte de nombreux types de mycorhizes de couleur, de taille et de morphologie variées. Cette diversité d'aspect dépend du partenaire fongique impliqué dans l'association avec le hêtre. Dans un article précédent (Prévost et Pargney, 1995), deux types de mycorhizes à lactaires (Lactarius blennius var viridis Fr et $L$ subdulcis SF Gray) ont été décrites. Elles présentent toutes deux une structure d'ectomycorhize mais chacune possède des caractéristiques particulières. Le manteau fongique, bien développé dans les deux cas, apparaît particulièrement protecteur pour les cellules racinaires dans l'association à $L$ blennius. Le réseau de Hartig est en revanche plus lâche que dans la mycorhize à $L$ subdulcis. Cette dernière, de structure ectomycorhizienne plus classique, présente la particularité de renfermer un autre champignon de type ascomycète.

Dans le présent travail, nous nous proposons de compléter cette étude comparative par les résultats obtenus après utilisation de techniques de cytochimie ultrastructurale. Cette approche doit permettre de caractériser les différentes interfaces des deux mycorhizes et d'identifier des composés de type polysaccharide et protéine impliqués, d'une part, dans les structures des mycorhizes et, d'autre part, dans les transferts entre les deux partenaires.

\section{MATÉRIEL ET MÉTHODES}

Les mycorhizes sont triées, prélevées et fixées pour la microscopie électronique à transmission selon des techniques précédemment décrites (Prévost et Pargney, 1995). Les coupes destinées à l'observation sont traitées suivant des méthodes adaptées à la cytochimie ultrastructurale.

\section{Test PATAg}

Ce test mis au point par Thiery (1967) met en évidence des polysaccharides en liaison $\alpha(1-4)$ ou $\beta(1-4)$. Les coupes, recueillies sur grilles en or, sont d'abord plongées à température am- biante dans l'acide periodique $1 \%$ pendant 30 minutes. Après rinçage par l'eau distillée, elles sont traitées par le thiocarbohydrazide à $0,2 \%$ dans l'acide acétique à $20 \%$ pendant 5 heures. Les grilles sont alors rincées par de l'acide acétique de concentration décroissante $(20,15,10$, $5 \%$ ), puis par de l'eau bidistillée à raison de deux bains de 5 minutes pour chaque. Les grilles sont traitées avec le protéinate d'argent à $1 \%$ pendant 30 minutes à l'obscurité. Après rinçage soigneux par l'eau bidistillée, les coupes sont observées en microscopie électronique à transmission.

\section{Réaction de Gomori-Swift}

La réaction de Swift (1968) permet de mettre en évidence les protéines riches en cystine.

Le réactif se prépare en mélangeant au moment de l'emploi dans $25 \mathrm{~mL}$ d'eau distillée (1), $25 \mathrm{~mL}$ de la solution A contenant $100 \mathrm{~mL}$ de méthanémine à $30 \%$ et $5 \mathrm{~mL}$ de nitrate d'argent à $5 \%, 5 \mathrm{~mL}$ de la solution $\mathrm{B}(2)$ renfermant $10 \mathrm{~mL}$ d'acide borique à $1,44 \%$ et $100 \mathrm{~mL}$ de Borax à $1,9 \%$.

Les coupes sont recueillies sur des grilles en or qui sont plongées dans la solution réactive à $45^{\circ} \mathrm{C}$ au bain-marie et à l'obscurité pendant 1 à 2 heures (selon le contraste souhaité).

Après plusieurs lavages soigneux à l'eau distillee, les grilles peuvent être observées en microscopie électronique à transmission.

\section{Marquage de la chitine par le complexe WGA-or colloïdal}

La lectine de blé (Wheat germ agglutinin) est une glycoprotéine qui se fixe spécifiquement sur les résidus $\mathrm{N}$-acétyl-D-glucosamines entrant dans la composition de la chitine. Par complexation avec l'or colloödal, la lectine fixée pourra être visualisée en microscopie électronique à transmission.

La technique s'effectue en plusieurs phases. Elle débute par la préparation de la suspension d'or colloïdal (Frens, 1973) permettant d'obtenir des particules d'or de diamètre de 15 à $17 \mathrm{~nm}$. $100 \mathrm{~mL}$ d'une solution à $0,01 \%$ d'acide tétrachloroaurique sont portés à ébullition. Au total, $4 \mathrm{~mL}$ d'une solution de citrate de sodium à $1 \%$ lui sont ajoutés. Après 5 à 10 minutes, le mélange, toujours à ébullition, prend une teinte rouge bordeaux. Après refroidissement, le $\mathrm{pH}$ acide de la solution est ajusté à 9,7 (point 
isoélectrique de la protéine) avec du carbonate de potassium $\left(\mathrm{K}_{2} \mathrm{CO}_{3}\right)$ à $0,02 \mathrm{M}$. Les prises d'essai du $\mathrm{pH}$ doivent être effectuées dans un tube à hémolyse avec $1 \mathrm{~mL}$ de suspension d'or et 3 gouttes de polyéthylèneglycol (PEG) 20000 à $1 \%$.

La préparation de la solution de lectine s'effectue en diluant $1 \mathrm{mg}$ de WGA dans $1 \mathrm{~mL} \mathrm{d'HCl}$ 2,5 M.

La complexation de la lectine par l'or nécessite tout d'abord de déterminer la quantité de lectine qui permet d'obtenir un complexe stable avecl'or (Roberts et al, 1983).

Une gamme de dilutions croissantes de lectine est réalisée à partir d'un tube de concentration en WGA de $0,1 \mathrm{mg} / \mathrm{L}$ (dilution de moitié jusqu'à la sixième dilution). Pour chaque dilution, un même volume d'or colloïdal est ajouté puis, une minute plus tard, $0,1 \mathrm{~mL}$ de $\mathrm{NaCl}$, dont le pouvoir électrolytique est utilisé pour la floculation de l'or libre. Celle-ci est visualisée par virage de la solution du rouge au bleu. La concentration où l'or est totalement couplé à la lectine correspond donc à celle du dernier tube ne virant pas et permet de déterminer la quantité de lectine saturant toutes les molécules d'or colloïdal.

Après mélange des deux solutions, le complexe formé est centrifugé à $15900 \mathrm{~g}$ pendant 50 minutes. Le sumageant, renfermant des molécules de lectine non associées, est éliminé. Le sédiment est alors repris par un mélange de tampon TRISchloride $20 \mathrm{mM}$ à $\mathrm{pH} 7$ contenant $0,04 \%$ de $\mathrm{PEG}$ 20000 et $0,02 \%$ d'azide de sodium.

Les coupes, recueillies sur grilles en or, sont mises à flotter pendant 30 minutes sur la solution WGA-or. Les coupes sont ensuite mises à rincer 2 fois 5 minutes sur du tampon TRIS-chloride puis sur de l'eau distillée. Le contraste est légèrement renforcé par double coloration acétate d'uranyle-citrate de plomb réduite à des durées de 5 minutes chacune.

Un témoin est réalisé en ajoutant à une solution WGA-or, une solution de N-acétyle-D-glucosamine $0,2 \mathrm{M}(1: 1)$. Les grilles sont mises à flotter pendant 30 minutes sur cette solution.

\section{RÉSULTATS}

\section{L'ectomycorhize à Lactarius blennius var viridis}

\section{Test PATAg}

Extérieurement, le manteau est limité par une substance fibrillaire peu réactive au test PATAg (fig 1). Les interfaces du manteau externe sont en revanche beaucoup plus marquées (fig 2). Cette réactivité diminue progressivement jusqu'au manteau interne (fig 3). Dans l'ensemble du manteau, le ciment est plus marqué que les parois fongiques. Les bandes polyphénoliques et le latex des laticifères sont denses aux électrons mais ne réagissent pas au test PATAg. Des rosettes de glycogène marquées par le test sont présentes dans de petites enclaves claires de cytosol, le long du plasmalemme (fig 3).

Dans le réseau de Hartig, le marquage des parois des deux symbiotes et du ciment est homogène (figs 4 et 5 ). La limite interne des parois des cellules corticales peut présenter un aspect granuleux (fig 5). Dans les hyphes, le test PATAg révèle des rosettes de glycogène regroupées en plages plus ou moins allongées (figs 4 et 5 ).

\section{Réaction de Gomori-Swift}

Le marquage de la substance périphérique est peu intense (fig 6). La réaction est plus importante dans les interfaces du manteau externe (fig 7) et du manteau interne (fig 8). Le marquage apparaît plus lâche dans le ciment que dans les parois fongiques. Celles-ci sont fortement réactives dans le manteau interne (fig 8). Les parois des laticifères réagissent comme les hyphes adjacentes (fig 8 ). Leur contenu n'est pas marqué par la réaction de Gomori-Swift.

Les interfaces du réseau de Hartig sont fortement réactives (fig 9). Les parois fongiques montrent un marquage différentiel, en deux strates: l'une, interne, est très réactive et elle est bordée d'un fin liseré plus clair. Le ciment et les parois des cellules corticales réagissent avec la même intensité et la limite entre les deux est difficile à distinguer. Le bord interne des cellules peut montrer parfois des accumulations granuleuses (fig 9). 


\section{Marquage par le complexe WGA-or colloïdal}

La substance périphérique est bien marquée par le complexe WGA-or colloïdal (fig 10). Dans le manteau, les parois fongiques de la zone externe ont un marquage plus important que celui des zones sousjacentes (figs 11 et 12). Le ciment ne réagit
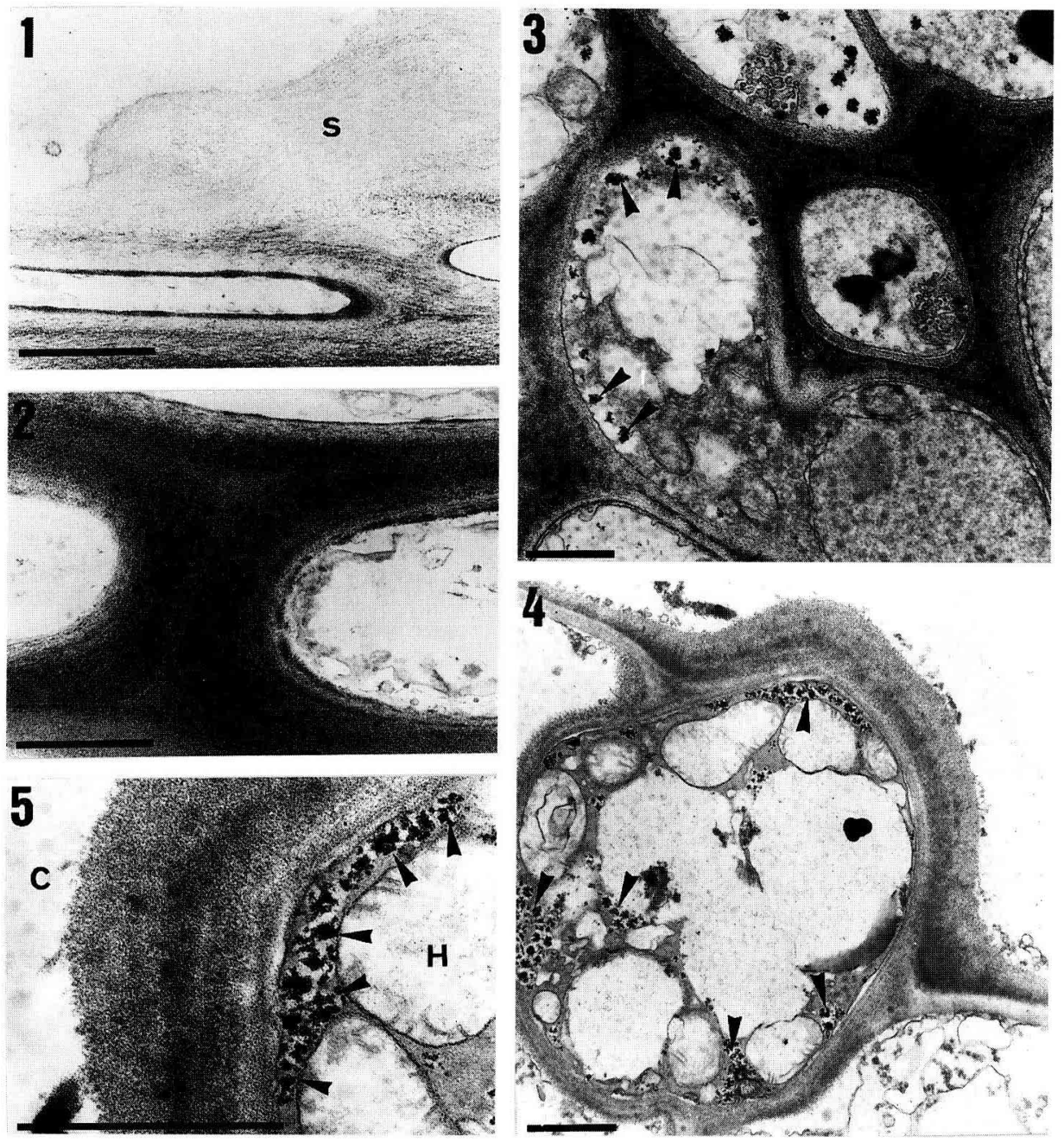

Figs 1-5. Mycorhize à L blennius. Test PATAg. 1. Substance mucilagineuse (s) limitant extérieurement le manteau (x 21000 ). 2. Interfaces du manteau externe (x 21000 ). 3. Hyphes du manteau interne renfermant du glycogène (flèches) $(x 13000)$. 4. Hyphe du réseau de Hartig avec rosettes de glycogène (flèches) $(x 13000)$. 5. Interface hyphe $(\mathrm{H})$-cellule corticale $(\mathrm{C})$ et détail des réserves de glycogène (flèches) (x 40000 ). (Les traits correspondent à $1 \mathrm{~m}$.) 
pratiquement pas (fig 12). Les parois des laticifères montrent un marquage comparable à celui des hyphes adjacentes (fig 13).

Dans le réseau de Hartig, les précipités d'or sont visibles au niveau des parois fongiques et non pas dans celles des cellules corticales (fig 14). Toutefois la strate la plus interne apparaît la plus réactive. Le ciment unissant les deux symbiotes n'est pas marqué. Les hyphes présentes à l'intérieur des cellules racinaires ont également une paroi réactive (fig 15).
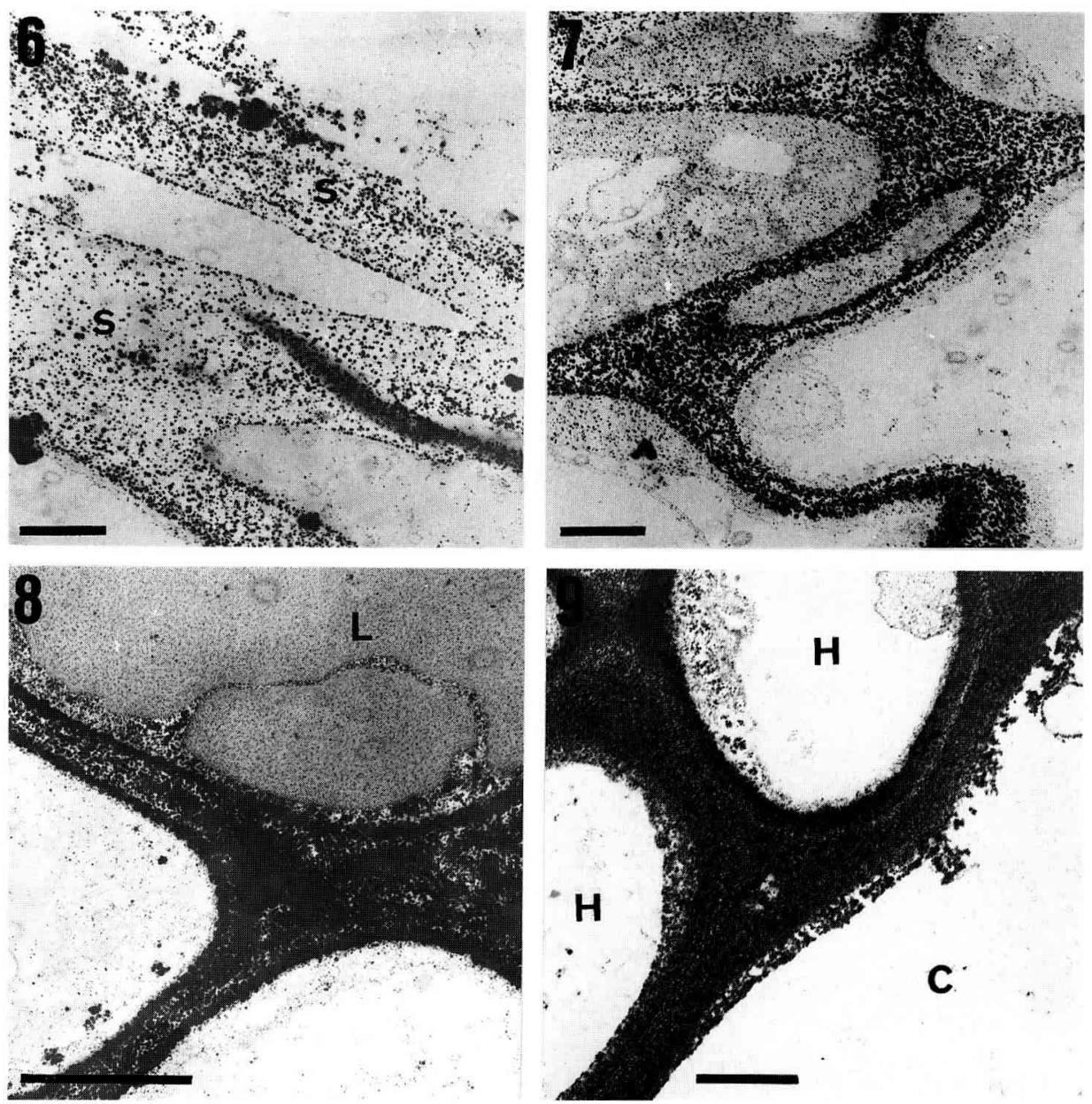

Figs 6-9. Mycorhize à L blennius. Réaction de Gomori-Swift. 6. Hyphes dans la substance périphérique $(s)(x 10000)$. 7. Hyphes du manteau externe (x 10 000). 8. Manteau interne avec laticifère (L) (x 20000$)$. 9. Hyphes du réseau de Hartig $(H)$ bordant une cellule corticale $(C)(x 12000)$. (Les traits correspondent à $1 \mathrm{~m}$.) 

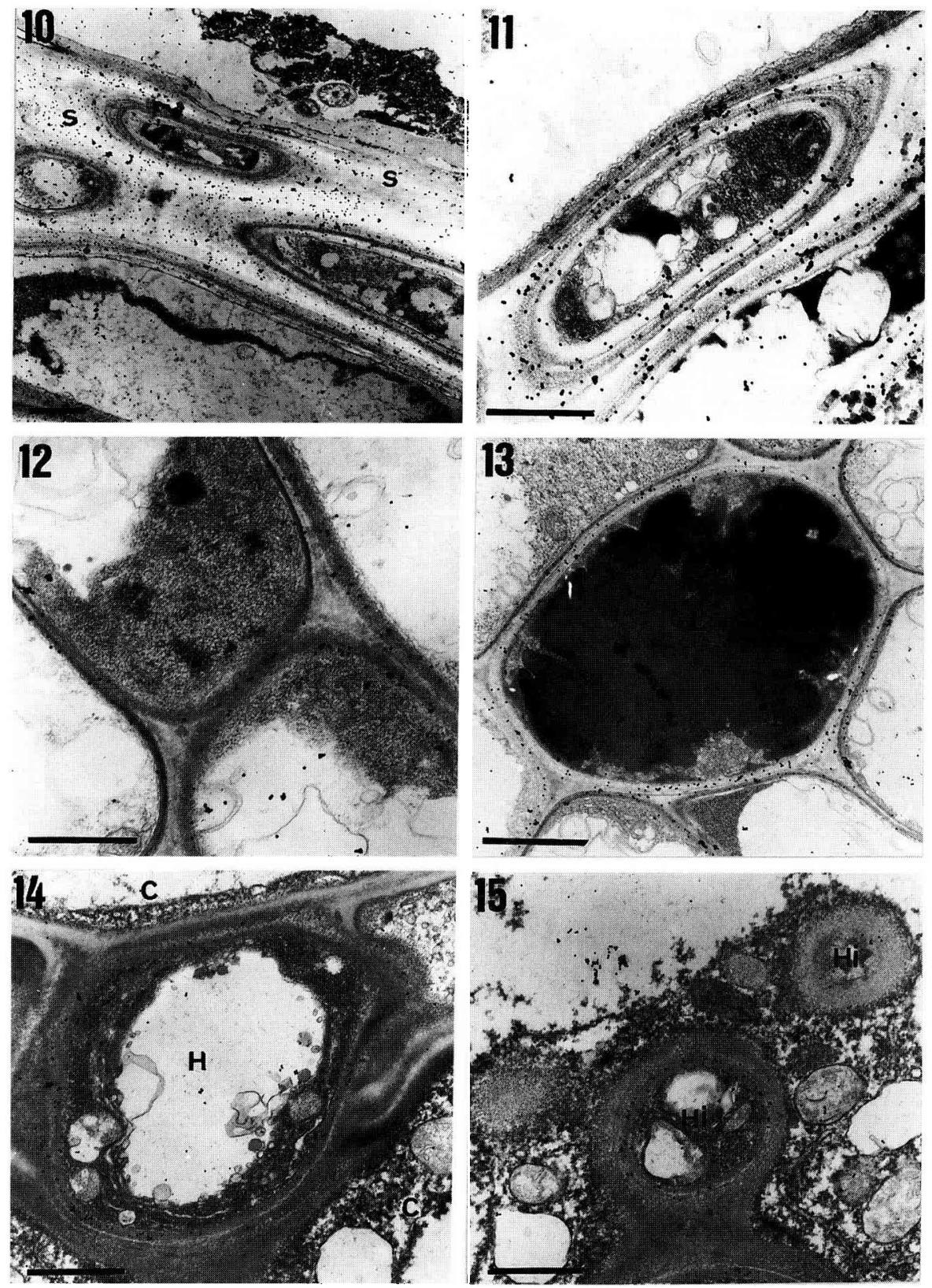

Figs 10-15. Mycorhize à $L$ blennius. Marquage WGA-or colloïdal. 10. Marquage de la substance (s) limitant le manteau externe (x 10000$)$. 11. Manteau externe (x 19000$)$. 12. Manteau interne (x 19 000). 13. Laticifère (L) du manteau interne (x 19000$)$. 14. Hyphe du réseau de Hartig $(H)$ et cellules corticales $(C)(x 17000)$. 15. Hyphes intracellulaires $(H i)$ dans une cellule corticale $(x 17000)$. (Les traits correspondent à $1 \mathrm{~m}$.) 


\section{L'ectomycorhize à Lactarius subdulcis}

\section{Test PATAg}

Dans le manteau externe, les parois fongiques sont bien marquées et le ciment très fortement contrasté (fig 16). Les parois et le ciment du manteau interne sont moins réactives (fig 17); une différence de contraste subsiste cependant entre ces deux structures. Les parois des laticifères réagissent comme celles des hyphes adjacentes. Des rosettes de glycogène sont visibles dans des zones claires (fig 17).

Dans le réseau de Hartig, les parois des deux symbiotes sont bien marquées (fig 18). Toutefois les strates les plus internes sont beaucoup plus contrastées dans les deux types de parois et un liseré clair entoure la strate dense des parois fongiques (fig 19). Le test PATAg révèle également des rosettes de glycogène souvent réparties le long du plasmalemme (fig 19).

Les hyphes de l'ascomycète associé à la mycorhize montrent une paroi stratifiée formée d'une couche externe très dense et d'une couche interne moins réactive (fig 20). Au cours de la pénétration intracellulaire de ces hyphes, les parois des deux partenaires sont étroitement en contact (fig 21). La paroi de la cellule hôte, bien que déformée, conserve en début de pénétration son intégrité. Au niveau de la zone de pénétration, la paroi fongique montre des strates supplémentaires plus marquées (fig 21).

\section{Réaction de Gomori-Swift}

Le manteau externe montre des interfaces fortement contrastées (fig 22). La réaction révèle trois couches pariétales d'épaisseurs différentes : i) une couche interne très marquée et épaisse, ii) une couche intermédiaire plus claire et large, iii) une couche externe fine et dense, qui borde le ciment fongique de réactivité comparable à celle de la couche intermédiaire. Dans le manteau interne, le ciment réagit très peu (fig 23) ; seules les couches pariétales fongiques externe et intermédiaire sont révélées.
Dans le réseau de Hartig, les parois des deux symbiotes sont réactives (fig 24) ; toutefois le liseré externe de la paroi fongique est moins marqué que la strate interne. Le ciment est difficilement différenciable des parois cellulaires de la racine. Celles-ci montrent une limite interne souvent granuleuse du fait de l'accumulation de dépôts d'origine cytoplasmique (fig 24). Des granulations marquées par le test de GomoriSwift sont également bien visibles dans des vésicules fongiques (fig 24).

L'intensité du marquage des hyphes intracellulaires de l'ascomycète est variable. La strate externe qui limite les hyphes est bien marquée (fig 25).

\section{Marquage par le complexe WGA-or colloïdal}

Les parois fongiques du manteau externe (fig 26), du manteau interne (fig 27) et du réseau de Hartig (fig 28) sont bien marquées. Les ciments ne réagissent pas.

Les précipités d'or sont également présents au niveau des parois de l'ascomycète. Le marquage est total sur toute l'épaisseur de la paroi lorsque les hyphes sont dans le manteau (fig 27). Il est limité à la strate pariétale interne lorsque les hyphes sont au niveau des cellules corticales (fig 29).

\section{DISCUSSION}

L'utilisation des techniques de cytochimie permet de caractériser les différentes interfaces dans les deux mycorhizes.

Les interfaces mycorhizosphère-manteau sont très différentes. Chez $L$ subdulcis, le manteau est directement en contact avec le sol et les hyphes les plus externes sont mortes et écrasées (Prévost et Pargney, 1995). En revanche, l'ectomycorhize à $L$ blennius est isolée du sol par du matériel mucilagineux qui est toutefois peu sensible au test PATAg et à la réaction de Gomori-Swift. La chitine, révélée par le marquage WGA-or colloïdal, est néan- 

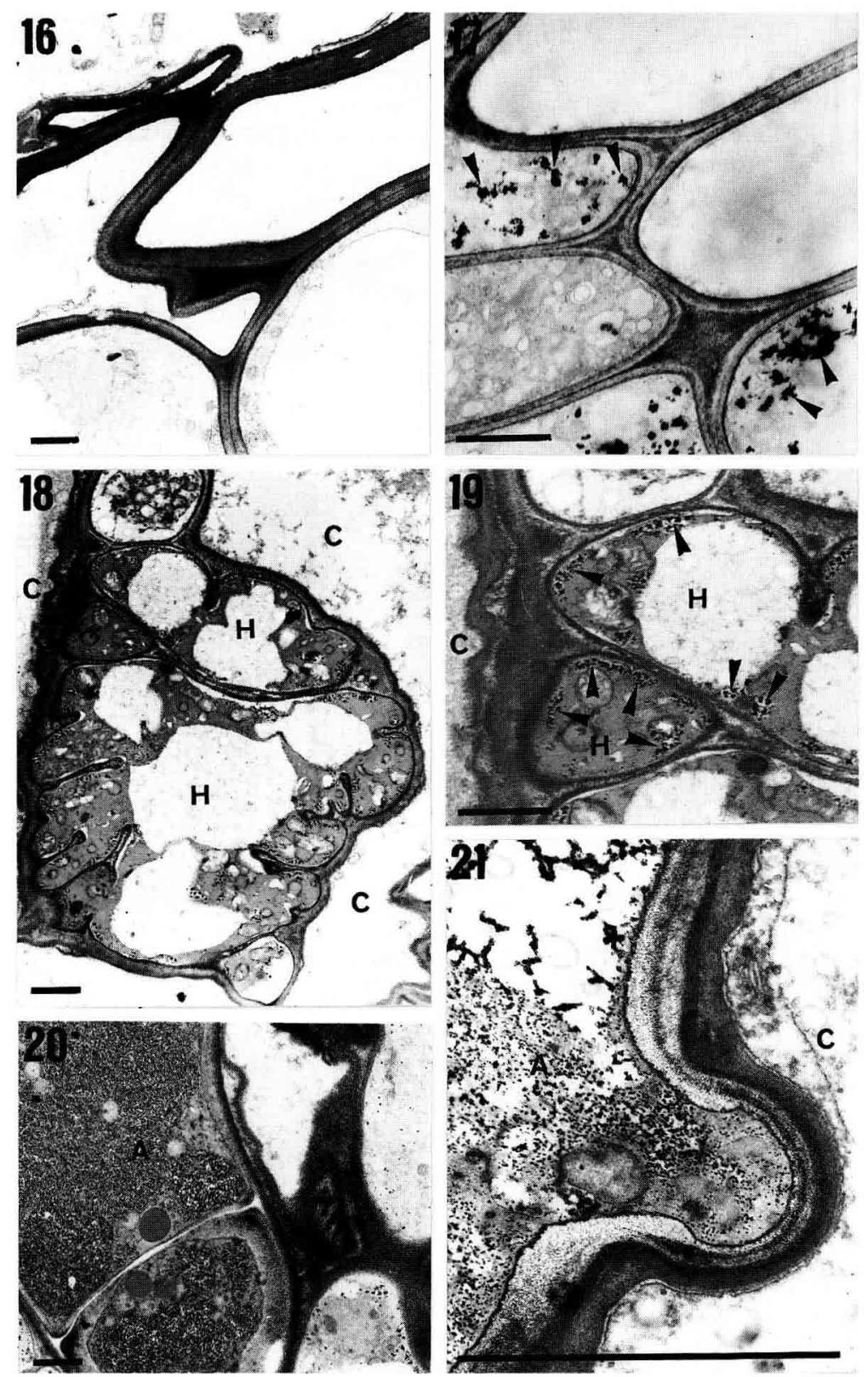

Figs 16-21. Mycorhize à $L$ subdulcis. Test PATAg. 16. Interfaces du manteau externe $(x 8000), 17$. Manteau interne avec grains de glycogène (flèches) $(x 16000)$. 18. Hyphes du réseau de Hartig $(H)$ bordant les cellules corticales (C) $(x 8000)$. 19. Détail de l'interface hyphes $(H)$-cellule corticale (C) et des réserves de glycogène (flèches) $(x 16000)$. 20. Hyphe d'ascomycète $(A)$ dans le manteau interne $(x 8000)$. 21. Pénétration intracellulaire d'une hyphe d'ascomycète $(A)$ dans une cellule corticale (C) (x 64000$)$. (Les traits correspondent à $1 \mathrm{~m}$.) 
moins bien représentée. Dans d'autres mycorhizes de lactaires, les deux cas ont été signalés : une couche mucilagineuse est présente chez $L$ flexuosus, $L$ piperatus, L chrysorrheus (Luppi et Gautero, 1967), $L$ volemus (Voiry, 1981) et $L$ vellereus (Luppi et Gautero, 1967 ; Agerer, 1987) ; les hyphes du manteau externe sont directement en contact avec le sol chez $L$ deterrimus (Münzenberger et al, 1986 ; Agerer, 1987 ) et $L$ picinus (Agerer, 1987).

Les interfaces dans le manteau sont constituées par les parois fongiques et le ciment interhyphal. Les tests cytochimi-
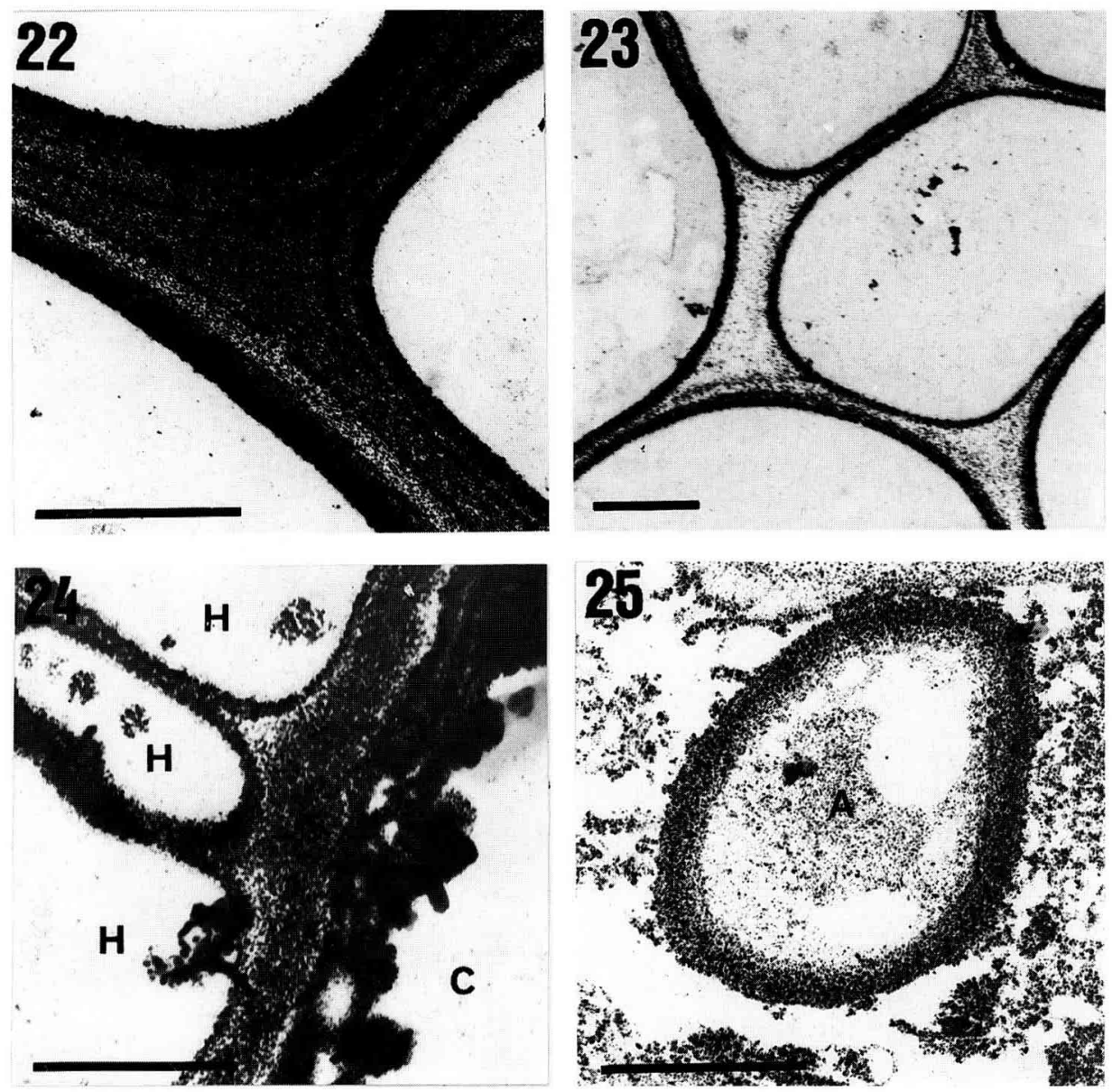

Figs 22-25. Mycorhize à $L$ subdulcis. Réaction de Gomori-Swift. 22. Interfaces du manteau externe (x 25000$)$. 23. Interfaces du manteau interne $(x 13000)$. 24. Interfaces hyphes du réseau de Hartig (H)-cellule corticale (C) (x 25000$)$. 25. Hyphe intracellulaire d'ascomycète (A) dans une cellule corticale ( $x 25000$ ). (Les traits correspondent à $1 \mathrm{~m}$.) 
ques montrent que les parois des deux lactaires sont pluristratifiées. Dans le manteau interne, elles apparaissent formées de deux strates, l'interne étant beaucoup plus sensible au test PATAg et à la réaction de Gomori-Swift. L'augmentation de l'épaisseur des parois dans le manteau externe se traduit par un accroissement du nombre des strates pariétales. La différence entre manteau externe et manteau interne se traduit également par une variation sensible des intensités de réaction au test PATAg : le marquage des parois fongiques diminue de l'extérieur vers l'intérieur du manteau. Cette différence a déjà été mentionnée dans d'autres ectomycorhizes (Pargney, 1991 ; Bawadikji, 1993). Elle doit correspondre à l'évolution des structures parié-
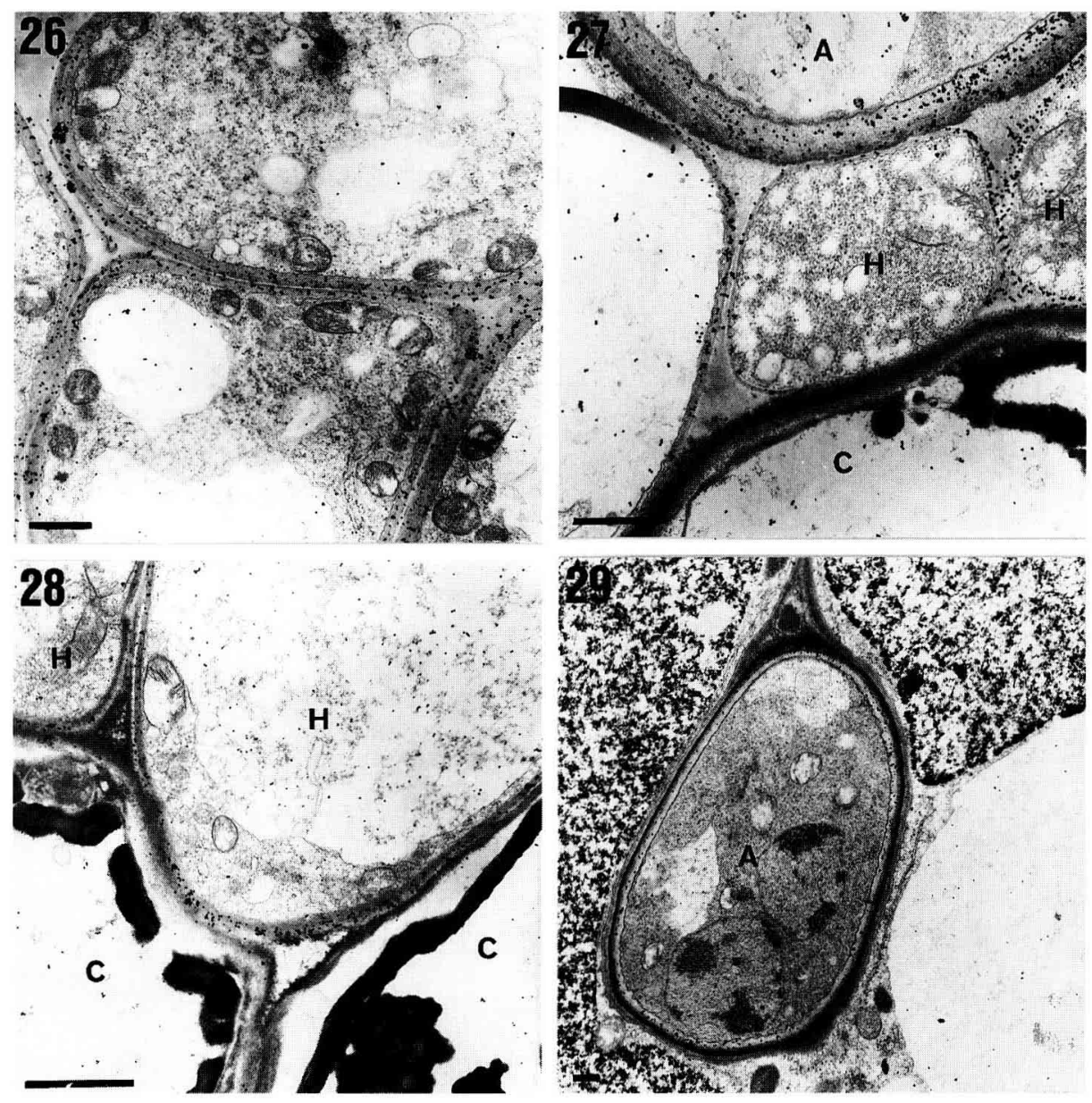

Figs 26-29 Mycorhize à $L$ subdulcis. Marquage WGA-or collö̈dal. 26. Hyphes du manteau externe $(x 10000)$. 27. Hyphes du manteau interne $(H)$ bordant une hyphe d'ascomycète $(A)$ et une cellule corticale (C) $(x 17000)$. 28. Réseau de Hartig $(H)$ et cellules corticales (C) (x 17 000). 29. Hyphe d'ascomycète $(A)$ dans une jeune cellule racinaire de l'apex de la mycorhize ( $x 4000)$. (Les traits correspondent à $1 \mathrm{~m}$.) 
tales qui dans le manteau externe subissent avec le ciment de profondes transformations aboutissant à l'établissement d'une interface plus résistante (Pargney, 1991).

Dans les deux mycorhizes, la WGA associée à l'or colloïdal marque exclusivement les parois fongiques. Des travaux précédents (Pargney 1990, 1991 ; Pargney et Gourp, 1991) ont déjà montré que la chitine n'intervient pas dans la constitution des ciments interhyphaux. Quel que soit le traitement cytochimique utilisé, ces ciments ne montrent d'ailleurs pas la même réaction que les parois fongiques adjacentes. Selon la mycorhize, le ciment montre une sensibilité différente à la réaction de GomoriSwift : positive chez $L$ blennius, négative chez $L$ subdulcis. Ainsi, il apparaît que chez $L$ blennius, les ciments du manteau et du réseau de Hartig sont composés en partie de glycoprotéines alors que chez $L$ subdulcis, la fraction protéique disparaît dans le manteau interne.

Les ciments interhyphaux des manteaux ont une origine fongique. Des études menées sur des champignons isolés ou lors des premiers stades d'infection ont pu mettre en évidence une couche d'aspect mucilagineux autour des parois des hyphes (Boutekrabt et Pargney, 1991). Cette couche participe à l'accrochage des hyphes entre elles pour former le manteau. L'édification de celui-ci à l'apex de la racine entraîne également l'incorporation de polyphénols issus des cellules de la coiffe racinaire (Pargney et Brimont, 1995). Ces polyphénols apparaissent alors dans le manteau sous forme de plages denses aux électrons entre les hyphes (Prévost et Pargney, 1995).

Au niveau du réseau de Hartig, les interfaces entre les cellules corticales et les hyphes réagissent fortement au test PATAg dans les deux mycorhizes. Les parois des cellules racinaires et la strate interne des parois fongiques sont également bien marquées par la réaction de Gomori-Swift. La couche interne est, comme dans le manteau interne, moins réactive. Comme dans le manteau, la chitine n'intervient pas dans la composition du ciment unissant les partenaires de la symbiose (Bonfante-Fasolo et al, 1987 ; Pargney, 1990 ; Pargney et Gourp, 1991).

II est communément admis que le champignon, au cours de son installation entre les cellules racinaires, démantèle et mobilise les composés pectiques de la lamelle moyenne (Debaud et al, 1981 ; Massicote et al, 1987 ; Pargney, 1990 ; Boutekrabt et Pargney, 1991). Une partie d'entre eux non utilisée par le champignon, peut être intégrée aux constituants d'origine fongique (Pargney, 1991). Le mode de croissance des hyphes formant le réseau de Hartig peut également intervenir au niveau de la composition du ciment. Celui de la mycorhize à $L$ subdulcis, dont les sections d'hyphes se succèdent sans espace libre (cf Prévost et Pargney, 1995), suggère un établissement progressif, par désolidarisation et lyse de la lamelle moyenne selon une action mécanique et une activité enzymatique du champignon. Au contraire, les digitations du réseau de Hartig de l'association à $L$ blennius (cf Prévost et Pargney, 1995) croissent en s'écartant les unes des autres. Les cellules corticales restent donc toujours localement en contact entre elles ; leur lamelle moyenne est, dans les zones non altérées, en continuité avec le ciment et la limite entre la lamelle moyenne et le ciment ne peut être mise en évidence ni par le test PATAg, ni par la réaction de Gomori-Swift. Les surfaces d'échanges entre le champignon et la plante hôte au niveau du réseau de Hartig apparaissent bien développées dans les deux mycorhizes. Dans l'association à $L$ subdulcis, le réseau unisérié entoure les cellules corticales sans laisser de contacts entre elles. Dans l'autre mycorhize, bien que le réseau soit discontinu, les nombreuses digitations de $L$ blennius offrent des surfaces d'échanges également importantes ; cependant, dans ce 
cas, une continuité symplasmique entre les cellules corticales subsiste.

La présence dans le cytoplasme de $L$ blennius et de $L$ subdulcis de rosettes de glycogène témoigne d'un apport glucidique d'origine racinaire. Les mitochondries présentes de part et d'autre de l'interface tant dans le cytoplasme fongique que dans les cellules corticales (cf Prévost et Pargney, 1995), révèlent une activité physiologique intense entre les partenaires symbiotiques. Toutefois le cytoplasme des cellules racinaires montre une dégénérescence plus précoce que celui des hyphes. II se transforme alors en des dépôts denses aux électrons le long des parois. Dans les cellules dégénérescentes, les limites des parois ne sont plus aussi précises ; ceci est notamment bien mis en évidence par le test PATAg et la réaction de Gomori-Swift. Cet aspect de la paroi a déjà été mentionné par Pargney et Gourp (1991) et Bawadikji (1993) sur d'autres ectomycorhizes.

L'interface que forme l'ascomycète avec les hyphes de lactaire ou les cellules racinaires est caractérisée par la présence d'une strate pariétale très dense limitant extérieurement les hyphes de l'ascomycète. Lorsque les hyphes sont à l'intérieur des cellules corticales, cette couche est bordée d'un fin liseré correspondant au plasmalemme de la cellule hôte. Celui-ci n'est pas rompu lors de la pénétration de l'hyphe dans la cellule. Les cellules racinaires ne semblent d'ailleurs pas réagir à cette pénétration : aucune modification pariétale n'est observée au niveau ou au voisinage de la zone de pénétration comme dans le cas des attaques par des champignons parasites (Griffiths, 1971 ; Delon et al, 1973) ; aucune accumulation polyphénolique importante ne se développe dans les cellules, au contact des hyphes de l'ascomycète. De telles accumulations ont été décrites dans des ectomycorhizes de synthèse de Boletus edulis lors de pénétrations intracellulaires anormales du champignon (Bawadikji, 1993).
La mise en évidence de la chitine par le complexe WGA-or colloïdal permet de révéler une évolution des structures pariétales des hyphes d'ascomycète : dans le manteau toute l'épaisseur de la paroi est marquée alors que chez les hyphes intracellulaires seule la couche pariétale interne l'est. II y a donc modification de la couche externe dense dont la chitine est vraisemblablement dépolymérisée lorsque l'hyphe devient intracellulaire. Des résultats similaires ont été obtenus avec un champignon endomycorhizien Gigaspora margarita (Grandmaison et al, 1988) : une réduction de la concentration en chitine des parois est observée entre les hyphes extra- et intracellulaires, mais aussi dans les hyphes intracellulaires elles-mêmes en fonction de la position de la ramification sur l'arbuscule.

En conclusion, les tests cytochimiques confirment la nature glycoprotéique des interfaces et l'absence de chitine dans les ciments des manteaux et des réseaux de Hartig des deux mycorhizes. Des variations peuvent cependant apparaître dans les intensités du marquage en fonction de la position des interfaces dans la mycorhize. Des différences apparaissent également entre les deux lactaires, comme par exemple la couche pariétale interne de $L$ subdulcis qui se révèle faiblement protéique dans le manteau interne et le réseau de Hartig.

Dans l'association à $L$ blennius, la couche mucilagineuse qui limite la mycorhize s'avère essentiellement de nature chitineuse. Elle correspond à une production fongique importante qui permet d'établir une interface manteau-mycorhizosphère nettement différenciée. Ce mucilage peut constituer une barrière efficace pour la mycorhize et assurer une protection tant mécanique que chimique. II pourrait également faciliter l'installation de la mycorhize en permettant une meilleure pénétration dans le sol. Son rôle vis-à-vis des microorganismes de la rhizosphère doit être aussi envisagé. 
Enfin, les tests cytochimiques révèlent la présence de glycogène dans le cytoplasme fongique essentiellement du réseau de Hartig. Ces accumulations le long du plasmalemme confirment les échanges entre les deux partenaires. Elles pourraient servir de marqueurs du flux de glucides transférés au champignon et permettraient de suivre les variations de ces apports au cours des différentes saisons.

\section{RÉFÉRENCES}

Agerer (1987) Colour Atlas of Mycorrhizae (R Agerer, ed). Einhorn-Verlag, Scwäbisch Gmünd

Bawadikji AH (1993) Essais de mycorhization contrôlée entre quatre cèpes et quelques essences forestières : techniques culturales et aspects ultrastructuraux. Thèse d'université, Énsa Montpellier

Bonfante-Fasolo P, Perotto S, Testa B, Faccio A (1987) Ultrastructural localization of cell surface sugar residues in ericoid mycorrhizal fungi by gold-labelled lectins. Protaplasma 139, 25-35

Boutekrabt A, Pargney JC (1991) Étude ultrastructurale de Tuber melanosporum Vitt en culture isolée et en association avec des vitroplants de Quercus ( $Q$ robur et $Q$ pubescens). Cryptogamie, Mycologie 12, 25-45

Debaud JC, Pépin R, Bruchet G (1981) Ultrastructure des ectomycorhizes synthétiques à Hebeloma alpinum et Hebeloma marginulatulum de Dryas octopetala. Can J Bot 59, 2160-2166

Delon R, Reisinger O, Mangenot F (1973) Étude aux microscopes photonique et électronique de racines de tomates var Marmande atteintes de maladie liégeuse. Ann Phytopathol 5, 151-162

Frens $\mathrm{G}$ (1973) Controlled nucleation for the regulation of the particle size in monodisperse gold solutions. Nature Phys Sci 241, 20-22

Grandmaison J, Benhamou N, Furlan V and Visser SA (1988) Ultrastructural localization of $\mathrm{N}$-acetylglucosamine residues in the cell wall of Gigaspora margarita throughout its life-cycle. Biol Cell 63, 89-100

Griffiths DA (1971) The development of lignitubers in roots after infection by Verticillium dahliae Kleb. Can J Microbiol 17, 441-444
Luppi AM, Gautero C (1967) Ricerche sulle micorrize di Quercus robur, $Q$ petraea et $Q$ pubescens in Piemonte. Allionia 13, 129-148

Massicotte HB, Peterson RL, Ackerley CA, Ashford AE (1987) Ontogeny of Eucalyptus pilularis-Pisolithus tinctorius ectomicorrhizae. Il. Transmission electron microscopy. Can J Bot 65, 1940-1947

Münzenberger B, Metzler B, Kootke I, Oberwinkler F (1986) Morphologische und anatomische Charakterisierung der Mykorrhiza Lactarius deterrimus-Picea abies in vitro. Z Mykol 52, 407-422

Pargney JC (1990) Essai de caractérisation cytochimique des structures de l'interface au niveau du réseau de Hartig dans l'association ectomycorhizienne entre la truffe (Tuber melanosporum Vitt) et le noisetier (Corylus avellana). Can J Bot 68, 27222728

Pargney JC (1991) Cytochimie ultrastructurale des interfaces présentes dans l'association ectomycorhizienne Tuber melanosporum/Corylus avellana. Cryptogamie, Mycologie 12, 47-61

Pargney JC, Brimont A (1995) Production of concentrated polyphenols by the root cap cells of Corylus associated with Tuber. ultrastructural study and element localization using electron energy loss spectroscopy and imaging. Trees 9, 149-157

Pargney JC, Gourp V (1991) Contribution à l'étude des mycorhizes de Pinus pinaster Soland : ultrastructure des associations obtenues avec deux basidiomycètes (Hebeloma cylindrosporum Romagn et Paxillus involutus Fr). Phytomorphology 41, 161173

Prévost A, Pargney JC (1995) Comparaison des ectomycorhizes naturelles entre le hêtre (Fagus sylvatica) et deux lactaires (Lactarius blennius var viridis et Lactarius subdulcis). I. Caractéristiques macroscopiques et cytologiques. Ann Sci For 52, 131-146

Roberts RL, Bowers B, Slater M, Cabib E (1983) Chitin synthesis and localization in cell division cycle mutants. Mol Cell Biol 3, 922-930

Swift JA (1968) The electron histochemistry of cystine containing proteins in thin transverse sections of human hair. J R Micr Soc 88, 449-460

Thiery JP (1967) Mise en évidence des polysaccharides sur coupes fines en microsocpie électronique. $J$ Microsc 6, 987-1018

Voiry H (1981) Classification morphologique des ectomycorhizes du chêne et du hêtre dans le nord-est de la France. Eur J For Path 11, 284-299 